\title{
A new hybrid protocol enabling to evaluate the pressure level of medical compression stocking in patients: "ex- vivo procedures".
}

\author{
D Rastel ${ }^{1,2}$, E Grenier ${ }^{3}$, B Lun ${ }^{2}$ \\ ${ }^{1}$ Selurl Philangio, Vascular Medicine, Grenoble, France \\ ${ }^{2}$ SCOTT, non-profit organization, Grenoble, France \\ ${ }^{3}$ SIGVARIS, Saint-Just-Saint-Rambert, France
}

submitted: Jul 30, 2017, accepted: Sep 24, 2017, EPub Ahead of Print: Oct 13, 2017, published: Dec 31, 2017

Conflict of interest: There is no conflict of interest regarding this work. D Rastel is a consultant for the medical device

industry with no exclusive agreement. E Grenier is an employee of Sigvaris company.

DOI: 10.24019/jtavr.30 - Corresponding author: Dr. Didier Rastel, d.rastel@wanadoo.fr, ,

(C) 2017 Fondazione Vasculab impresa sociale ONLUS. All rights reserved.

\begin{abstract}
Introduction Medical compression treatment using stockings exerts a pressure to the vascular system which represents the therapeutic dose. This pressure is indirectly measured according to heavy laboratory tests based on calibrated artificial leg markings. The objectives of this work were to elaborate and test a new dynamometer which allows direct measurement of pressure from how the stocking is applied by the patient and whatever the leg curvature radius are.

Method A portable mini dynamometer (Modyn) that reproduces the testing in textile laboratories was elaborated. To test the reliability of this device four sizes, each size available in three lengths, of anti-thromboembolic medical compression stockings were measured at the ankle level using both the Modyn and the reference textile laboratory dynamometer. All the measurements were blindly performed. The measurement procedure followed the G 30102 B French norm.

Results On a total of 72 measurements, the explained variance between both methods was $\mathrm{r}^{2}=0.92$ and the correlation coefficient $r=0.96$. The intra-operator repeatability of the Modyn provided a mean value of 22.12 mmHg, SD 0.74.

Conclusion This portable, easy to use, pressure measurement device using the dynamometer principle, is a reliable field tool that should be used in future clinical trials to measure more precisely what the patients treated

by compression stockings really receive as therapeutic pressure.

Keywords Medical compression stockings, Pressure measurement, Dynamometer, Interface pressure, Lower limb

\section{Introduction}

Compression therapy is a leading treatment of superficial venous disorders and can be applied to patients by using bandages, medical compression stockings (MCS) and sustained or intermittent air pressure therapy. Bandages are more frequently used as a first line treatment in severe cases such as ulcers. Notwithstanding the efficacy, the applied pressure to the diseased leg is mainly linked to the operator's skills due to the Laplace's law type of pressure. The pressure profile applied by air pressure therapy devices, accurately controlled by the machine, is based on the BoyleMariotte's law: $\mathrm{PV}=\mathrm{MkT}(\mathrm{P}=$ pressure, $\mathrm{V}=$ volume, $\mathrm{T}$ = temperature and $\mathrm{M}=$ mass, $\mathrm{K}=$ coefficient $)^{1}$, which is the principle of the arterial pressure cuffs. In spite of the benefits in pressure level accuracy, air pressure therapy is by far less ambulatory than bandages or stockings. MCS, being ambulatory, are ideally supposed to have a controlled pressure profile on the patient's legs.
\end{abstract}


MCS are classified in four compression classes, corresponding to different therapeutic dosages. They are prescribed to patients according to their degree of pathologies ${ }^{2}$. Numerous national and international recommendations for prescriptions have been issued by medical societies. One of them, widely considered as the most relevant statement on compression therapy, highlights the limits of our knowledges on the compression treatment ${ }^{3}$. In many countries, MCS are certified through a normalized process. In France, independent technical laboratories use the French norms AFNOR G30.102B to evaluate the MCS pressure ${ }^{4}$. This procedure, called "in-vitro" procedure, firstly requires to don and mark the MCS on a normalized Hoheinstein wooden leg (with dimensions corresponding to the MCS size) and secondly to place the MCS on a standard and heavy dynamometer to stretch the MCS, simulating the leg dimensions ${ }^{5}$. The results will indicate the amount of pressure exerted at specific points such as the ankle, the calf and the thigh.

In spite of its perfect adequacy with the industrial process for MCS manufacturing, that procedure is not satisfactory for clinical purposes. Indeed, in spite of the almost perfect characteristics of the MCS, following rigorous qualification and certification systems based on the national standards of many countries, the question of the effective pressure, as really applied to human legs, is still pending.

To better evaluate the true pressure applied to the patient's legs, numerous protocols labelled "in-vivo" protocols have been developed ${ }^{6}$. Those cheap, fast and easy-to handle devices and procedures consisted in placing a small pressure sensor between the skin and the MCS fabric.

Unfortunately, the results depended mostly on the exact location of the sensor on the leg (local limb curvature) and less on the apparatus accuracy. This protocol is known as highly operator dependent ${ }^{7}$. Subsequently, it is almost impossible to compare the pressure level mentioned on the packaging and being the official characteristics of the MCS, and the pressure level measured using the "in-vivo" procedure.

Moreover when for instance, the calf muscle contracts, the curvature (local limb radius), varies in such a proportion (values at certain points of the leg) that the "in-vivo" technique would rather measure the muscle anatomical properties than the intrinsic MCS properties themselves ${ }^{8}$. Some authors call that the "working-resting pressure". Therefore, "in-vivo" and "invitro" measurements can be compared only when "in-vivo" sensors are placed on a human leg on a very precise place which has a curvature value corresponding to the circumference of the wooden leg used for the "in-vitro" testing ${ }^{9}$.

For example, to measure the "in-vivo" pressure on an ankle of a $23 \mathrm{~cm}$ circumference the sensor must be placed on an area where curvature radius is of $3.67 \mathrm{~cm}$. In addition, the investigator must be equipped with a device enabling the measurement of the local limb radius. In order to address these questions, a new procedure is being elaborated and a new device is designed aiming at providing "in-vivo" results which would be comparable to the normalized "invitro" figures. We name this technique "ex-vivo" protocol.

\section{Method}

Instrumentation / Tool. This new device is a portable mini-dynamometer that reproduces the "in-vitro" testing usually performed by using a usual (non-portable) dynamometer in textile laboratories. It is a non-destructive testing method since the patients can get back to their stockings after the "ex-vivo" procedure is completed. The specifications of this device are provided in Figure 1. We call it "Modyn", an abbreviation of Mobile Dynamometer. In a nutshell, this hybrid "ex-vivo" method allows the investigator to obtain figures of pressure close to the true effective pressure independently without the curvature radius value.

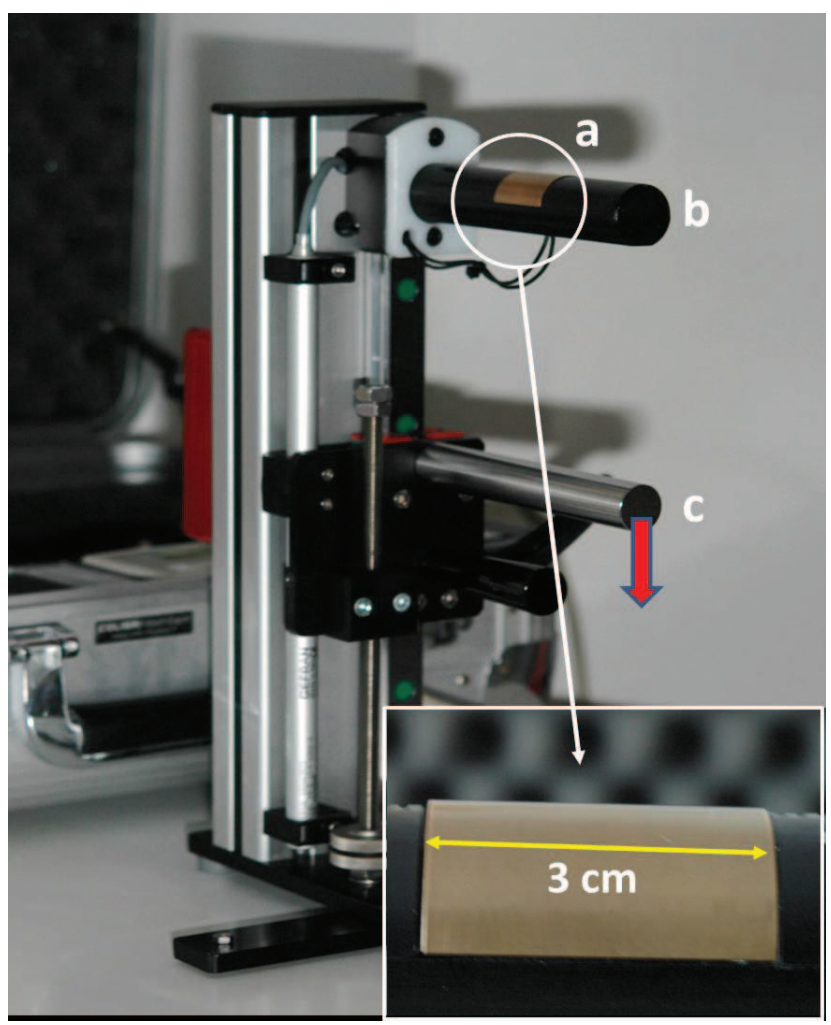

Figure 1 - The MObile DYNamometer (MODYN) device. a) the force sensor, $b$ ) the fixed part and c) the mobile part. Enlarged, the force detection zone of $3 \mathrm{~cm}$ length. 


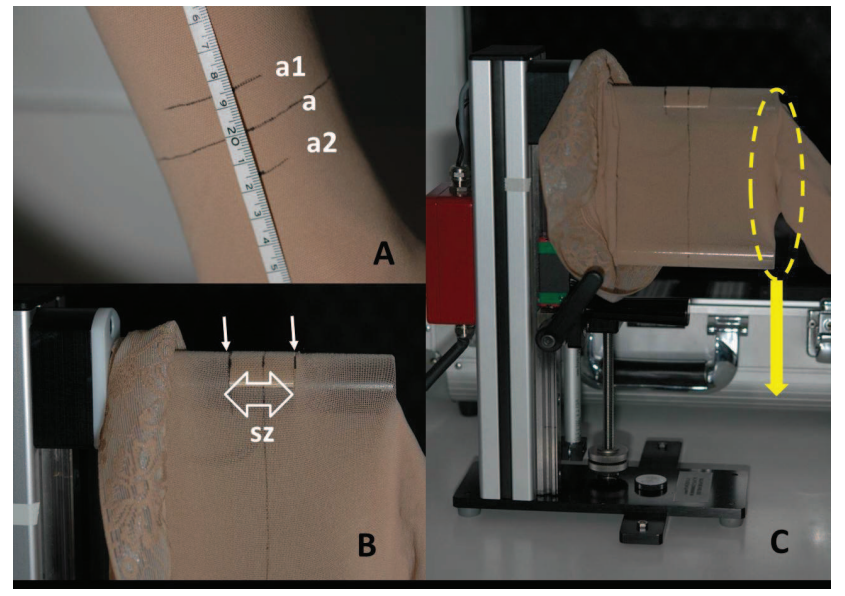

Figure 2 - Principle of ex-vivo measurements. A. Marks tracing on MCS when worn on leg; a, circumference line, a1, above mark at $1.5 \mathrm{~cm}$ and a2 below mark at $1.5 \mathrm{~cm}$ from the circumference line. B. Placement of marked MCS on Modyn and positioning the marks (white arrows) on the sensor zone (sz). C. The fabric is stretched at the related circumference using the mobile part of the Modyn (yellow arrow).

The methodology for the pressure measurement is performed in four steps.

1) firstly, the patient puts the MCS on his or her leg as he or she is usually doing without any interference/training from the investigator.

2) secondly, the investigator measures the limb circumference at the point where he wants to know the amount of pressure exerted by the MCS and traces a line around the limb at that point. At an equal distance of 1.5 $\mathrm{cm}$ from this circumference line, two marks are proximally and distally traced on the stockings. Those $3 \mathrm{~cm}$ (distance between the 2 marks) correspond exactly to the sensor zone of the Modyn (Figure 2).

3) thirdly, the patient wears off the stocking. After what, the stocking is positioned on the Modyn. A gentle massage is performed in order to homogenously distribute the MCS fabric; the 2 marks performed at the second step must be placed at the edges of the force sensor of the Modyn.

4) finally, the MCS is stretched at an elongation corresponding to the defined circumference value at the second step. The obtained results are compared to the data issued from the measurements performed on a usual dynamometer in a textile technical laboratory using the equipment required by the NF G30-102B norms.

\section{Protocol}

The objective of the present task is to compare the data obtained from the Modyn to the data issued from the Usual Dynamometer in textile laboratory (UD) which is cited in the NF G30-102B norm. The same MCS are involved; they are tested at the same marked areas and stretched within the same circumference. Only the attachment of the MCS fabric to the force sensor differs from Modyn to UD. The tested stockings were antithromboembolic medical compression stockings (ATE) manufactured by SIGVARIS (Saint-Just-Saint-Rambert, France). Four sizes, T1 (size 1) to T4 (size 2), of this same product range were involved; each size is available in three lengths (which are short, normal and long. Both items of each pair were tested. In total twenty four MCS were evaluated and twenty four measurements collected.

The stockings were donned on the leg of one volunteer having a $22 \mathrm{~cm}$ ankle circumference. The evaluation process followed the procedure detailed in the previous paragraph. Additionally, the range of measurements was extended to two other dimensions, 21 $\mathrm{cm}$ and $23 \mathrm{~cm}$ so as to consolidate the validity of the Modyn. In total seventy two measurements from Modyn were compared to seventy two measurements from UD.

Modyn measurements were carried out by a qualified investigator having adequate skills regarding the device. Then, the stockings were sent to a textile laboratory in order to be measured on a UD; they were carried out by a technician well-trained on the UD measurement techniques. All the measurements were blindly performed; the operators didn't know the size nor the compression class of the MCS. They were only requested to test the fabric at the marked area which was the ankle zone, by stretching them at $21 \mathrm{~cm}, 22 \mathrm{~cm}$ and $23 \mathrm{~cm}$ circumferences.

\section{Statistics}

The descriptions used basic statistical parameters: mean and standard deviation for quantitative variables, and frequency and percentage for qualitative variables. To build a relationship between the two quantitative variables, the statistic correlation was used and the Pearson linear correlation coefficient (r) calculated.

\section{Results}

If we consider only the "ex-vivo" results which represent twenty four measurements on all sizes and lengths, stretched to $22 \mathrm{~cm}$ related to the involved patient having $22 \mathrm{~cm}$ ankle circumference, the mean pressure obtained on Modyn was $19 \mathrm{mmHg}$, SD 5.52 and the mean pressure obtained by UD was $18 \mathrm{mmHg}$, SD 5.52 (Graph $1)$. The explained variance between both methods was $r^{2}=$ 0.93 and the correlation coefficient $r=0.96$ (Graph 2).

If we compare the overall seventy two figures (all items tested at $21 \mathrm{~cm}, 22 \mathrm{~cm}$ and $23 \mathrm{~cm}$ ), the explained variance was $r^{2}=0.92$ and the correlation coefficient $r=0.96$ (Graph 3). 


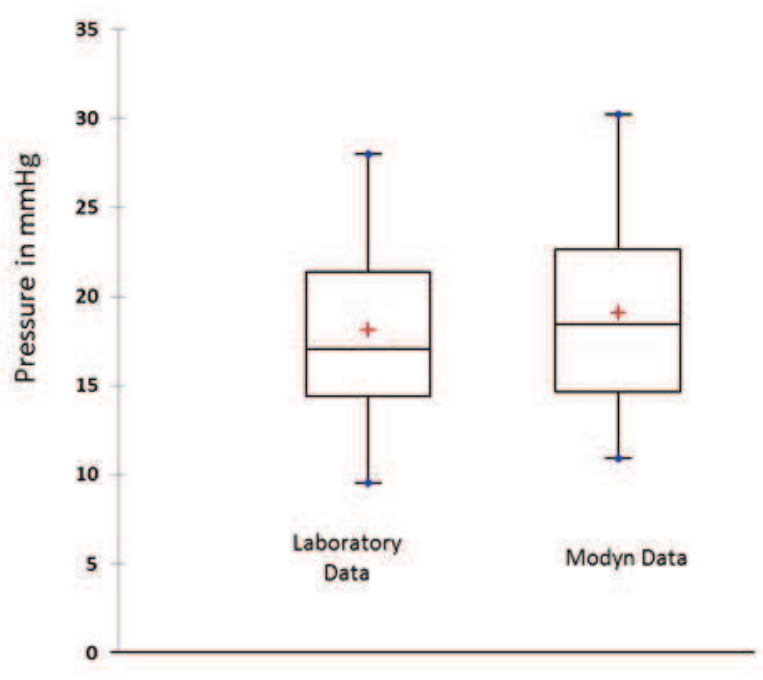

Graph 1 - Comparison of pressure values laboratory dynamometer (left) versus Mobile dynamometer (right) on 24 measurements of all sizes at $22 \mathrm{~cm}$ of ankle circumference. Mean, $S D, 5$ and 95 percentiles.

The intra-operator repeatability of the UD within five measurements carried out on same above item (size $2 \mathrm{~N}$ stretched at $22 \mathrm{~cm}$ ankle circumference) provided a mean value of $21.9 \mathrm{mmHg}$, SD 0.68. The intra-operator variability of the Modyn within five measurements carried out on size $2 \mathrm{~N}$ stretched $22 \mathrm{~cm}$ ankle circumference provided a mean value of $22.12 \mathrm{mmHg}$, SD 0.74 .

Last but not least, another set of five measurements performed by a third operator on Modyn carried out on the item mentioned above (size $2 \mathrm{~N}$ stretched $22 \mathrm{~cm}$ ankle circumference) provided a mean value of $22.6 \mathrm{mmHg}, \mathrm{SD}$ 0.34 . Therefore, the variability inter operator on Modyn is minor.

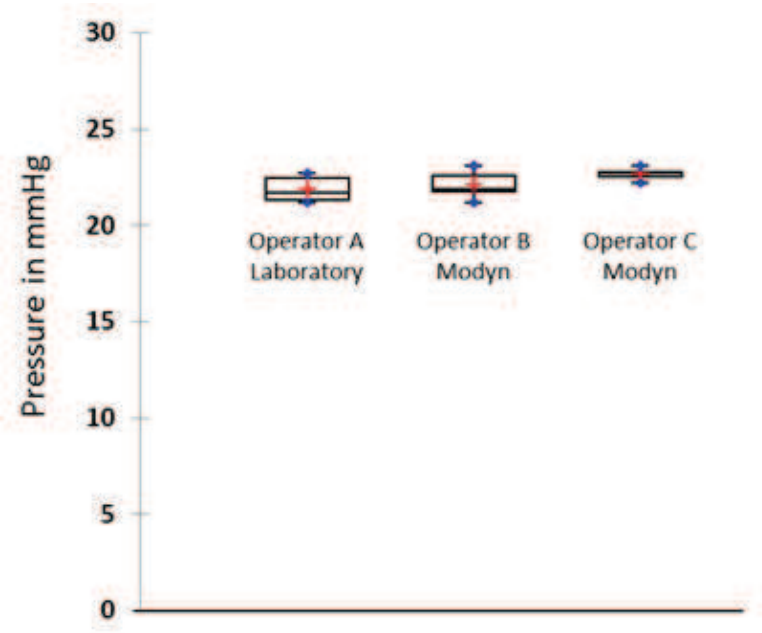

Graph 2 - Intra operator repeatability of measurements on Modyn. Mean, SD, 5 and 95 percentiles.

\section{Discussion}

Medical compression treatment involving stockings (MCS) is based on the application of an interface pressure above the skin and all along the lower limb; the pressure being partially transmitted to the venous system. Its role, in superficial and/or deep venous insufficiencies, is to counterbalance the intravascular venous hypertension caused by venous disorders. As a consequence, the transmural pressure equilibrium is reduced to a more normal level. The MCS effect is then a question of pressure amount.

MCS are ranged in four classes according to the pressure exerted on the ankle. That supposes that the ankle circumference of the patient who will wear a given MCS, would correspond to the size. That also underlines the importance of the marking process of the area to be stretched by the dynamometer. The norms recommend the marking task to be performed on wooden Hohenstein standard leg or Marking board.

Unfortunately, very little is known about the pressure effectively applied to patients in published trials on compression therapy. We only know the theoretical mean pressure exerted by the stocking used in the trial, but nothing beyond that. Besides the true morphological status of the patient's leg with pressure exerted along his or her leg, and not only at the ankle level, are not precisely known.

The new procedure described here-after permits to fill this gap and provides a field device to measure the pressure on a given patient's leg in the clinical trials. According to our new procedure, the marking process is performed directly on the patient's leg when the MCS is already worn. By consequence, the process will measure how the patient, once the MCS put on, had spread the threads along his or her leg.. The "ex-vivo" procedure allows to evaluate the effective pressure exerted by a given MCS on a given patient's leg, donned in a given way.

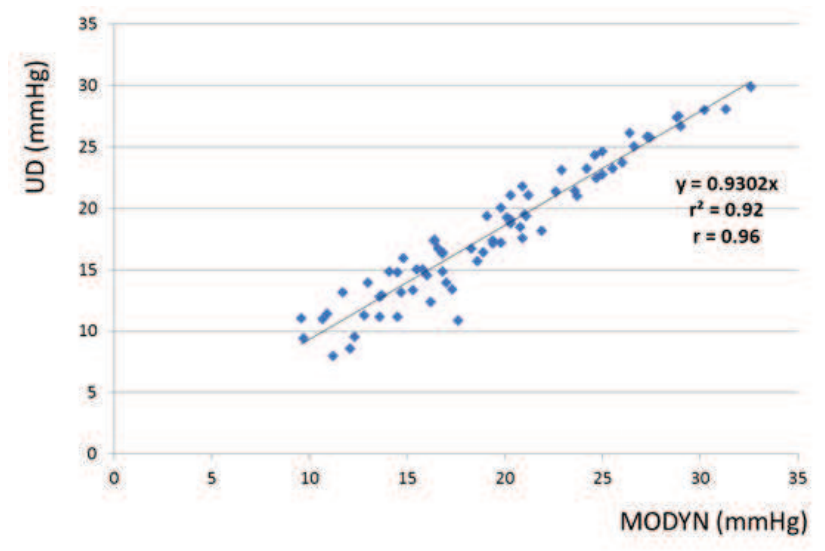

Graph 3 - Overall correlation of pressure measurements, UD (Usual Dynamometer) versus MODYN (MObile DYNamometer). 
Consequently, it provides the closest value to the true and effective pressure exerted by the stocking on the patient's leg. That is the innovative part of the "ex-vivo" method. The results demonstrate that the Modyn device is not only a practical device but is also a reliable tool as its results are perfectly correlated to the data issued from the currently used method in textile laboratories (UD) which complies with national norms.

According to the results mentioned above, it would be interesting to use this "ex-vivo" procedure with mobile dynamometer or usual laboratory dynamometer, in clinical studies involving MCS. It would allow us to know the effective interface pressure, in other words, the dosage of the compression treatment without any interference from the local limb radius.

\section{References}

1) Partsch H. Intermittent pneumatic compression in immobile patients. Int Wound J. 2008;5(3):389-97.

2) Benigni JP, Gobin JP, Uhl JF, Cornu-Thénard A. Utilisation quotidienne des bas médicaux de compression. Recommandations cliniques de la Société Française de Phlébologie. Phlébologie 2009;62(3):95-102

3) Partsch H. Compression therapy: clinical and experimental evidence. Ann Vasc Dis. 2012;5(4):416-22.

4) Articles de bonneterie - Détermination de la pression de contention. Norme AFNOR NF G30-102B. Octobre 1986. www.afnor.org.

5) Quality Certification Mark 387/1 (RAL-GZ 387/1). Medical compression hosiery. www.hohenstein.in.
Nevertheless, it must be highlighted that the main parameter causing the interface pressure variability is more related to the donning action, namely how the patient stretches the stocking on his leg, than the performance of the instrument in use. The adequacy of the MCS size with the patients' leg dimensions is also less important than we thought.

\section{Conclusion}

The Modyn, which is a portable- easy to use- pressure measurement device using the dynamometer principle, is a reliable field tool that should be used in future clinical trials to measure more precisely what the patients really receive as therapeutic pressure.

6) Partsch H. Interface pressure and stiffness of ready made compression stockings: Comparison of in vivo and in vitro measurements. J Vasc Surg 2006;44:809-14.

7) Strolïn A. Biophysical Characteristics of Medical Compression Stocking Phebologie 2007;36:197-204.

8) Uhl JF, Lun B. Action physique et effet physiologique des BMC; Signification de la notion "Pression travail-Pression repos" [Physical and Physiological Effects of Medical Compression Stockings: Notions of Resting-Working Pressures]. Phlébologie 2008; 61: $29-41$

9) W. Blättler W, Lun B, Uhl JF, Bringer Y. Determinants of pressure exerted by medical compression stockings. Phlebologie 2007;36:237-44. 